\title{
Quantum-field Theories as Representations of a Single*-Algebra
}

\author{
A. Raab \\ Hyltans Sjöväg 12, 43539 Mölnlycke, Sweden \\ ${ }^{*}$ Corresponding Author: andreas.raab.mail@web.de
}

Copyright (C)2013 Horizon Research Publishing All rights reserved.

\begin{abstract}
We show that many well-known quantum field theories emerge as representations of a single ${ }^{*}$-algebra. These include free quantum field theories in flat and curved space-times, lattice quantum field theories, Wightman quantum field theories, and string theories. We prove that such theories can be approximated on lattices, and we give a rigorous definition of the continuum limit of lattice quantum field theories.
\end{abstract}

Keywords Quantum Field Theory, String Theory, Borchers-uhlmann Algebra, Lattice Field Theory, Continuum Limit

\section{Introduction}

The Wightman distributions play a fundamental role in Wightman quantum field theories (Wightman QFTs) [1]. The reconstruction theorem demonstrates that knowledge of the Wightman distributions is sufficient to obtain a unique Wightman QFT. In particular, the Wightman distributions define a state of a Borchers-Uhlmann (BU) algebra, and the associated Wightman QFT is obtained as a representation of a BU-algebra from that state [2,3]. Interestingly, interacting as well as non-interacting Wightman QFTs emerge as representations of the same BU algebra.

Although the Wightman axioms provide a remarkably successful framework in Minkowski space-time, they cannot be generalized to curved space-times in a straightforward manner. This is one of the motivations to study QFT in an algebraic framework, and substantial progress has been achieved with this approach in recent years. An overview of the field can be found in Refs. $[4,5]$, and we will discuss more details of those achievements in Sec. 5. However, the approach taken in Ref. [4] yields an interesting aspect that results from the generalization of the axiomatic approach to curved space-times. Starting point of the axiomatic approach in Ref. [4] is a free *-algebra, Free $(M)$, of quantum fields on a background structure, $M$, which, amongst others, refers to a globally hyperbolic space-time. The quantum-field algebra, $A(M)$, is obtained by factoring Free $(M)$ by a set of relations, in which coefficients of an operator-product expansion (OPE) play a fundamental role, i.e. there exists a ${ }^{*}$-homomorphism, $\pi$ : Free $(M) \rightarrow A(M)$, which essentially is defined by properties of the OPE coefficients. The set of states of the theory, $S(M)$, is further constrained to support the OPE, positivity, and a microlocal spectrum condition. However, if we construct a representation of $A(M)$ from a state $\omega \in S(M)$, then we basically obtain a representation of Free $(M)$ with respect to the state $\omega \circ \pi$. The set of states of the QFT can therefore also be seen as a subset of the set of states over Free $(M)$, which is obtained by appropriate constraints. Quite different QFTs can therefore emerge as representations of the same *-algebra, Free $(M)$, and we note that Free $(M)$ plays an analogous role as the BU-algebras in Wightman QFTs. We pick up this idea and explore it in a more general approach in this paper.

In Sec. 2.1, we introduce the general setting, in which we define the terms test-function space, Q-map, and Qtheory. Q-maps are mathematical generalizations of quantum fields that lack specific physical properties, and Q-theories are corresponding generalizations of QFTs. We use Q-maps and Q-theories as purely technical devices to construct appropriate *-algebras, from which QFTs arise as representations. In particular, as it is the case for quantum fields in Wightman QFTs, for example, the image of a Q-map is a set of operators in a ${ }^{*}$-algebra, and a Q-theory is the continuous representation of the polynomial algebra generated by the operators in the image of a Q-map. In Sec. 2.2 we further define in which sense a Q-theory emerges from a representation of a ${ }^{*}$-algebra (i.e. the polynomial algebra 
generated by a Q-map), and in Sec. 2.3 we introduce a Q-map whose polynomial algebra, $\mathcal{A}_{0}$, can be represented in any Q-theory, so that the Q-theory emerges from that representation. We also prove that Q-theories can be approximated on lattices, and we give a rigorous definition of the continuum limit. In the remaining part of the paper we show that many well-known QFTs are Q-theories that emerge as representations of $\mathcal{A}_{0}$. These are the central results of this paper.

We discuss in Sec. 3 the applicability of the approach to hermitian scalar Wightman QFTs, and in Sec. 4 we discuss free scalar QFTs and Dirac QFTs in curved space-times. In Sec. 5, we continue with the discussion of perturbatively interacting quantum fields in curved space-times, and we also relate to recent developments in algebraic QFT in more detail, c.f. Refs. $[4,5]$. In Sec. 6, we consider string theories and lattice QFTs, and we discuss the continuum limit of lattice QFTs more concretely.

\section{Q-maps and Q-theories}

\subsection{General setting}

Let us begin with the definition of some general terms. In Wightman QFTs, quantum fields are defined as operatorvalued distributions over a test-function space. We choose a similar approach:

1. A conjugation, $C$, on a complex vector space, $V$, is an antilinear map satisfying $C^{2}=1$ and $C(a v+b w)=$ $\bar{a} C(v)+\bar{b} C(w)$ for all $a, b \in$ and all $v, w \in V$.

2. Let $V_{1}$ and $V_{2}$ be vector spaces with conjugations $C_{1}$ and $C_{2}$. A c-homomorphism, $h: V_{1} \rightarrow V_{2}$, is a vector-space homomorphism that is compatible with the conjugations, i.e. $h \circ C_{1}=C_{2} \circ h$.

3. A test-function space is a complex separable locally-convex Hausdorff topological vector space on which a conjugation is defined.

4. A Q-map is a complex-linear map from a test-function space into a ${ }^{*}$-algebra, $\Phi: V \rightarrow P(\Phi)$, where $P(\Phi)$ is the polynomial algebra generated by the set of operators $\Phi(V) \cup\{1\} \cdot{ }^{1} \Phi$ further satisfies $\Phi(f)^{*}=\Phi(C f)$ for all $f \in V$.

5. A Q-theory is a pair $(\Phi, \omega)$, where $\Phi$ is a Q-map, $\omega$ is a state over $P(\Phi)$, and, for all $n \in$,

$$
\omega\left(\prod_{m=1}^{n} \Phi(\cdot)\right)
$$

is a multi-linear continuous functional on $V^{n}$.

We note that we restrict test-function spaces to be separable and that larger test-function spaces will be considered in a subsequent paper. Moreover, we will show in subsequent sections that quantum fields typically are Q-maps and that QFTs typically are Q-theories. We note however that Q-maps and Q-theories lack specific physical properties, like commutation relations, for example, and we emphasize that they serve a purely technical purpose in this paper. In particular, the above definitions are not intended as a set of axioms for QFTs.

Let us further elaborate on our definitions. An example of a test-function space is Schwartz space, $S\left({ }^{n}\right)$. Complex conjugation is given in $S\left({ }^{n}\right)$ by

$$
C(a f+b g)(x)=\bar{a} \bar{f}(x)+\bar{b} \bar{g}(x)=\bar{a}(C f)(x)+\bar{b}(C g)(x) .
$$

Moreover, for any locally-convex topological vector space, $V$, a test-function space can be constructed as follows. Let $\bar{V}$ denote the corresponding complex conjugate vector space (c.f. appendix A.2 in Ref. [6]), and let $j: V \rightarrow \bar{V}$ denote the natural anti-linear bijection. The set $\{j(B): B$ open in $V\}$ is a locally-convex Hausdorff topology on $\bar{V}$. Let $W=V \oplus \bar{V}$ be equipped with the product topology of $V \times \bar{V}$, and define the conjugation $C(f, g)=\left(j^{-1}(g), j(f)\right)$, then $W$ is a test-function space.

A test-function space, $V$, always has an associated Q-map, which can be constructed as follows. Let

$$
\mathcal{A}_{V}=\bigoplus_{n=0}^{\infty} V^{\otimes n}
$$

denote the tensor algebra of $V$. For the sake of notational convenience, we denote an element in $V^{\otimes n}$ by

$$
v_{1} \otimes v_{2} \otimes \ldots \otimes v_{n}=\bigotimes_{m=1}^{n} v_{m}
$$

\footnotetext{
${ }^{1}$ We note that if the field operators satisfy CCRs or CARs, then $P(\Phi)$ is generated by $\Phi(V)$ alone.
} 
Moreover, we define the involution

$$
\begin{aligned}
C^{(n)}\left(\bigotimes_{m=1}^{n} v_{m}\right) & =\bigotimes_{m=1}^{m} C\left(v_{n-m+1}\right) \quad\left(v_{m} \in V\right) \\
\left(a_{n}\right)^{*} & =\left(C^{(n)} a_{n}\right) \quad\left(\left(a_{n}\right) \in \mathcal{A}_{V}\right),
\end{aligned}
$$

so that $\mathcal{A}_{V}$ is a (non-commutative) ${ }^{*}$-algebra. We further define the complex-linear Q-map, $\Phi_{V}: V \rightarrow \mathcal{A}_{V}$, by

$$
\Phi_{V}(v)=(0, v, 0,0, \ldots) \quad(v \in V),
$$

then $P\left(\Phi_{V}\right)=\mathcal{A}_{V}$. Note that for $\left.V=S{ }^{n}\right), \mathcal{A}_{V}$ is a BU-algebra.

Multiple Q-maps can be combined into a single Q-map as follows. Let $\Phi_{i}: V_{i} \rightarrow P\left(\Phi_{i}\right)(i \in I)$ be at most countably many Q-maps, for which multiplication and addition of operators are defined. Define

$$
V=\bigoplus_{i} V_{i}, \quad C\left(v_{i}\right)=\left(C_{i} v_{i}\right)
$$

and equip $V$ with the product topology, i.e. $V$ is a subspace of $\prod_{i} V_{i} . V$ is a complex separable locally-convex Hausdorff topological vector space, i.e. $V$ is a test-function space. Define further the map

$$
\Phi\left(\left(f_{i}\right)\right)=\sum_{i} \Phi_{i}\left(f_{i}\right), \quad \Phi\left(\left(f_{i}\right)\right)^{*}=\sum_{i} \Phi_{i}\left(f_{i}\right)^{*},
$$

then $\Phi$ is a $Q$-map.

Proposition 1: Let $\Phi: V \rightarrow P(\Phi)$ be a Q-map, let $W$ be a test-function space, and let $h: W \rightarrow V$ be a chomomorphism, then $\Phi \circ h$ induces a ${ }^{*}$ homomorphism, $\pi: P\left(\Phi_{W}\right) \rightarrow P(\Phi)$, so that $\Phi \circ h=\pi \circ \Phi_{W}$.

Proof: $\Phi \circ h$ is a complex-linear function from $W$ into $P(\Phi)$ that uniquely extends to an algebra homomorphism, $\pi$, from the tensor algebra $\mathcal{A}_{W}$ of $W$ to $P(\Phi)$ by the universal property of the tensor algebra. Let $C_{V}$ denote the conjugation on $V$, and let $C_{W}$ denote the conjugation on $W . \pi$ further is a *homomorphism:

$$
\pi\left(\left(a_{n}\right)\right)=a_{0}+\sum_{n} \bigotimes_{m=1}^{n} \Phi\left(h\left(w_{m, n}\right)\right) \quad\left(\left(a_{n}\right) \in \mathcal{A}_{W}, a_{n}=\bigotimes_{m=1}^{n} w_{m, n} \in W^{\otimes n}, w_{m, n} \in W\right),
$$

and

$$
\begin{aligned}
\pi\left(\left(a_{n}\right)\right)^{*} & =\bar{a}_{0}+\sum_{n} \bigotimes_{m=1}^{n} \Phi\left(h\left(w_{n-m+1, n}\right)\right)^{*}=\bar{a}_{0}+\sum_{n} \bigotimes_{m=1}^{n} \Phi\left(C_{V}\left(h\left(w_{n-m+1, n}\right)\right)\right)= \\
& =\bar{a}_{0}+\sum_{n} \bigotimes_{m=1}^{n} \Phi\left(h\left(C_{W} w_{n-m+1, n}\right)\right)=\pi\left(\left(a_{n}\right)^{*}\right) .
\end{aligned}
$$

In particular, $(\Phi \circ h)(w)^{*}=\left(\pi \circ \Phi_{W}\right)(w)^{*}(w \in W)$.

Definition 1: Let $\Phi_{i}: V_{i} \rightarrow P\left(\Phi_{i}\right)$ be Q-maps $(i=1,2)$. $\Phi_{1}$ is a core for $\Phi_{2}$ if there exists a continuous chomomorphism, $h: V_{1} \rightarrow V_{2}$, and a ${ }^{*}$ homomorphism, $\pi: P\left(\Phi_{1}\right) \rightarrow P\left(\Phi_{2}\right)$, so that $h\left(V_{1}\right)$ is dense in $V_{2}$ and that $\Phi_{2} \circ h=\pi \circ \Phi_{1}$. If $h$ is surjective, then $\Phi_{2}$ is a quotient of $\Phi_{1}$.

Corollary 1: Let $\Phi: V \rightarrow P(\Phi)$ be a Q-map, then $\Phi$ is a quotient of $\Phi_{V}$.

Proof: The identity map, id : $V \rightarrow V$, is a surjective continuous c-homomorphism, so that proposition 1 yields $\Phi=\pi \circ \Phi_{V}$

As mentioned above, QFTs typically are Q-theories. Let us assume $\Phi_{2} \circ h=\pi \circ \Phi_{1}$ as in definition 1, i.e. $\Phi_{1}$ is a core for $\Phi_{2}$, and let $\left(\Phi_{2}, \omega_{2}\right)$ be a QFT. $\omega_{1}=\omega_{2} \circ \pi$ is a state on $P\left(\Phi_{1}\right)$, and $\left(\Phi_{1}, \omega_{1}\right)$ is a Q-theory. We argue in the following that $\left(\Phi_{1}, \omega_{1}\right)$ and $\left(\Phi_{2}, \omega_{2}\right)$ essentially yield the same quantum theory.

\subsection{Quantum theories from Q-map cores}

Let $(\Phi, \omega)$ be a Q-theory. $\omega$ induces the representation of $P(\Phi)$ on a pre-Hilbert space, $D_{\omega}$, by the GNS construction (for *-algebras). Elements in $D_{\omega}$ are given by equivalence classes of operators in $P(\Phi)$. The representation is commonly denoted by $\left(H_{\omega}, \pi_{\omega}, \Omega_{\omega}\right)$, where $H_{\omega}$ is the completion of $D_{\omega}, \pi_{\omega}$ is a ${ }^{*}$-homomorphism, and $\Omega_{\omega}$ is the unit vector corresponding to the unity operator, 1. Expectation values of operators in $P(\Phi)$ are given by

$$
\left\langle u_{[a]}, \pi(b) u_{[c]}\right\rangle=\left\langle u_{[a]}, u_{[b c]}\right\rangle=\omega\left(a^{*} b c\right) \quad(a, b, c \in P(\Phi)) .
$$


The continuity property in our definition of a Q-theory further guarantees that

$$
\left\langle u, \prod_{i_{1}}^{n} \Phi\left(f_{i}\right) v\right\rangle
$$

defines a complex-linear multi-continuous functional for all $u, v \in D_{\omega}$. This is satisfied in scalar Wightman QFTs, for example.

However, let $\tau_{\omega}$ denote the locally-convex topology generated by the set of semi-norms, $\left\{\left\|\pi_{\omega}(\cdot) u\right\|: u \in D_{\omega}\right\}$, on $P(\Phi)$. Let us further assume that $V$ contains a subset, $V_{s}$, so that $\pi_{\omega}(\Phi(f))$ is essentially self-adjoint for all $f \in V_{s}$ and that $P(\Phi)$ is generated by $\Phi\left(V_{s}\right)$. We call such a Q-theory regular. Let $\mathcal{A}$ be the $\mathrm{C}^{*}$-algebra generated by the set $\left\{\exp \left(i \pi_{\omega}(\Phi(f))\right): f \in V_{s}\right\}$, then $\pi_{\omega}(P(\Phi))^{\prime}=\mathcal{A}^{\prime}$, i.e. the commutants agree, and $\pi_{\omega}(P(\Phi))^{\prime \prime}=\mathcal{A}^{\prime \prime}$. $\mathcal{A}^{\prime \prime}$ may be seen as the algebra of observables of the state $\omega$, which contains all projection-valued measures that are relevant in the respective theory (c.f. Def. 2.6.3 in Ref. [7]). If the set $\left\{\Phi(f): f \in V_{s}\right\}$ is irreducible, then the set $\left\{\exp \left(i \pi_{\omega}(\Phi(f))\right): f \in V_{s}\right\}$ is also irreducible, and $\mathcal{A}^{\prime \prime}$ equals the set of linear-bounded operators on $H_{\omega}$. However, by von-Neumann's density theorem, $\mathcal{A}$ is dense in $\mathcal{A}^{\prime \prime}$ with respect to the weak operator topology, and the restriction of any operator in $\mathcal{A}^{\prime \prime}$ to $D_{\omega}$ is the $\tau_{\omega}$-limit of a net of polynomials of operators in $\Phi(V)$.

Let $\tilde{\Phi}$ be a core for $\Phi$, i.e. there exists a continuous c-homomorphism, $h$, and a *homomorphism, $\pi$, so that $\Phi \circ h=\pi \circ \tilde{\Phi}$, then $\pi(P(\tilde{\Phi}))$ is dense in $P(\Phi)$ with respect to the $\tau_{\omega}$-topology. In particular, each operator in $\pi_{\omega}(P(\Phi))$ is the strong-graph limit of a net of operators in $\pi_{\omega} \circ \pi(P(\tilde{\Phi}))$. If $(\Phi, \omega)$ is regular, then $\pi_{\omega} \circ \pi(P(\tilde{\Phi}))^{\prime \prime}=\mathcal{A}^{\prime \prime}$.

Proposition 2: Let $(\Phi, \omega)$ be a Q-theory, and let $\tilde{\Phi}$ be a core for $\Phi$, then there exists a ${ }^{*}$-homomorphism, $\pi$, so that each operator in $\pi_{\omega}(P(\Phi))$ is the strong-graph limit of a net of operators in $\pi_{\omega} \circ \pi(P(\tilde{\Phi}))$.

Let us re-formulate proposition 2 into a looser statement: $(\Phi, \omega)$ and $(\tilde{\Phi}, \omega \circ \pi)$ essentially yield the same theory, which emerges from a representation of $P(\tilde{\Phi})$.

\subsection{A universal Q-map}

Let $V_{0}$ be the vector space of cofinite complex sequences,

$$
V_{0}=\bigoplus_{n=1}^{\infty}
$$

and define the conjugation

$$
C_{0}\left(\left(c_{n}\right)\right)=\left(\bar{c}_{n}\right) \quad\left(\left(c_{n}\right) \in V_{0}\right)
$$

Lemma 1: Let $V$ be a test-function space. There exists a c-homomorphism, $h: V_{0} \rightarrow V$, so that $h\left(V_{0}\right)$ is dense in $V$. If $V$ is finite-dimensional, then $h$ is surjective.

Proof: Let $\left\{f_{n}\right\}$ be a countable, dense subset of $V$, let $W$ be the linear span of $\left\{f_{n}\right\}$, and let $C$ denote the conjugation on $V$. Let further $\left\{e_{n}\right\}$ be a maximal linear-independent subset of

$$
\left\{\left(f_{n}+C\left(f_{n}\right)\right\} \cup\left\{i\left(f_{n}-C\left(f_{n}\right)\right)\right\}\right.
$$

then $\left\{e_{n}\right\}$ is a basis of $W$. Note that if $W$ is finite-dimensional, then $W$ is closed and thus $W=V$. Moreover, the function

$$
h\left(\left(c_{n}\right)\right)=\sum_{n=1}^{\operatorname{dim} W} c_{n} e_{n} \quad\left(\left(c_{n}\right) \in V_{0}\right)
$$

is a vector-space homomorphism between $V_{0}$ and $W$, which is compatible with the conjugations,

$$
C\left(h\left(\left(c_{n}\right)\right)\right)=\sum_{n} \bar{c}_{n} e_{n}=h\left(C_{0}\left(\left(c_{n}\right)\right)\right) .
$$

Let $\mathcal{V}$ be the class of test-function spaces, and for $V \in \mathcal{V}$ let $h_{V}: V_{0} \rightarrow V$ be a c-homomorphism so that $h\left(V_{0}\right)$ is dense in $V$. If $V=V_{0}$ as sets, and if the conjugation on $V$ is $C_{0}$, then we choose $h_{V}=$ id. We equip $V_{0}$ with the initial topology generated by the set $\left\{h_{V}^{-1}(B): B\right.$ open in $\left.V, V \in \mathcal{V}\right\}$. Note that this is the weakest topology for which all functions $h_{V}(V \in \mathcal{V})$ are continuous.

Lemma 2: $V_{0}$ is a test-function space.

Proof: We first note that a function $f: Z \rightarrow V_{0}$ is continuous if and only if $h_{V} \circ f$ is continuous for all $V \in \mathcal{V}$. Let $f_{c, W}$ denote multiplication with $c \in$ on a vector space, $W . f_{c, V_{0}}$ is continuous since $h_{V} \circ f_{c, V_{0}}=f_{c, V} \circ h_{V}$, and since scalar 
multiplication is continuous on $V$ for all $V \in \mathcal{V}$. Let $g_{W}(a, b)=a+b$ denote the addition function on a vector space, $W . g_{V_{0}}$ is continuous since $\left(h_{V} \circ g_{V_{0}}\right)(a, b)=g_{V}\left(h_{V}(a), h_{V}(b)\right)=h_{V}(a)+h_{V}(b)$, and since addition is continuous on $V$ for all $V \in \mathcal{V}$. Let further $a, b \in V_{0}, a \neq b$, let $V=V_{0}$ as sets, let $V$ be equipped with the topology induced by the semi-norms $p_{m}\left(\left(c_{n}\right)\right)=\left|c_{m}\right|\left(\left(c_{n}\right) \in V\right)$, and let $C_{0}$ be the conjugation on $V$, then $V$ is a test-function space. The topology of $V$ is contained in the topology of $V_{0}$ by definition of $V_{0}$. Since $V$ is Hausdorff, there exist open sets $A$ and $B$ in $V$ so that $a \in A, b \in B$, and $A \cap B=\emptyset$. $V_{0}$ is therefore a Hausdorff space since $A$ and $B$ are also open in $V_{0}$. For each $V \in \mathcal{V}$ let $\mathcal{B}_{V}$ denote a neighborhood base of 0 of balanced, convex, absorbing sets, and let $\mathcal{B}_{V_{0}}$ be the set of finite intersections of sets in $\left\{h_{V}^{-1}(B): B \in \mathcal{B}_{V}, V \in \mathcal{V}\right\} . \mathcal{B}_{V_{0}}$ is a neighborhood base of 0 . Due to linearity, $h_{V}^{-1}(B)$ is a balanced, convex, and absorbing set for all $B \in \mathcal{B}_{V}$ and all $V \in \mathcal{V}$. Let $B \in \mathcal{B}_{V_{0}}, B=C_{1} \cap \ldots \cap C_{n}$, and let $a \in V_{0}$. B is balanced and convex. For each $C_{i}(1 \leq i \leq n)$ there exists a $t_{i}>0$ so that $a \in t C_{i}$ if $t \geq t_{i}$. Let $t_{0}=\max \left\{t_{1}, \ldots, t_{n}\right\}$, then $a \in t B$ if $t \geq t_{0}$. $B$ is therefore absorbing, and $V_{0}$ is locally convex. Moreover, $V_{0}$ is the union of countably many finite-dimensional spaces,

$$
V_{0}=\bigcup_{n} V_{0, n}, \quad V_{0, n}=\left\{\left(c_{m}\right) \in V_{0}: c_{m}=0 \forall m>n\right\}
$$

Since each $V_{0, n}$ is finite-dimensional, the respective subspace topologies are equivalent to the Eucledian topologies, which entails that each $V_{0, n}$ is separable. Let $W_{0, n}$ be a countable, dense subset of $V_{0, n}$, then $\bigcup_{n} W_{0, n}$ is a countable, dense subset of $V_{0}$.

Let $\Phi_{0}: V_{0} \rightarrow \mathcal{A}_{0}$ be the Q-map associated with $V_{0}$. Lemma 1 and proposition 1 yield the following theorem.

Theorem 1: Let $\Phi: V \rightarrow P(\Phi)$ be a Q-map, then $\Phi_{0}$ is a core for $\Phi$. If $V$ is finite-dimensional, then $\Phi$ is a quotient of $\Phi_{0}$.

Considering proposition 2, $\Phi_{0}$ actually is a universal Q-map, since any Q-theory basically is a Q-theory of $\Phi_{0}$, which emerges from a representation of $P\left(\Phi_{0}\right)$.

Theorem 2: Let $\omega$ be a state over $P\left(\Phi_{0}\right)$, then $\left(\Phi_{0}, \omega\right)$ is a Q-theory.

Proof: We need to show that, for all $n \in$,

$$
\omega^{(n)}=\omega\left(\prod_{m=1}^{n} \Phi_{0}(\cdot)\right)
$$

is a multi-linear continuous functional on $V_{0}^{n}$. Let $F$ be the set of linear functions from $V_{0}$ to . For each $f \in F$ we define the semi-norm $p_{f}(v)=|f(v)|\left(v \in V_{0}\right)$. The set of semi-norms, $\left\{p_{f}\right\}_{f \in F}$, defines a locally-convex Hausdorff topology on the set $V_{0}$. Let $V_{0}^{F}$ denote the corresponding topological space. We choose $C_{0}$ as conjugation on $V_{0}^{F}$, so that $V_{0}^{F}$ is a test-function space. Each $f \in F$ is continuous when considered as a function from $V_{0}^{F}$ to . Due to the definition of the test-function space $V_{0}$, each open set in $V_{0}^{F}$ is also open in $V_{0}$, so that each $f \in F$ is also continuous when considered as a function from the test-function space $V_{0}$ to . Hence, $\omega^{(n)}$ is continuous in each argument, and therefore it is continuous on $V_{0}^{n}$.

Let $S_{0}$ be the set of states over $P\left(\Phi_{0}\right)$. Each $a \in P\left(\Phi_{0}\right)$ defines a linear functional on $S_{0}$ by $l_{a}(\omega)=\omega(a)$ for $\omega \in S_{0}$. The corresponding set of semi-norms, $p_{a}(\omega)=\left|l_{a}(\omega)\right|=|\omega(a)|$, defines a topology on $S_{0}$, and a net $\left(\omega_{i}\right)$ in $S_{0}$ converges to an $\omega \in S_{0}$ with respect to that topology, if $\lim _{i} \omega_{i}(a)=\omega(a)$ for all $a \in P\left(\Phi_{0}\right)$.

Let $V_{0, n}=\left\{\left(c_{m}\right) \in V_{0}: c_{m}=0 \forall m>n\right\}$. We will argue in Sec. 6.1 that $V_{0, n}$ is the test-function space of a quantum system with $n$ degrees of freedom. Such quantum systems typically are considered in lattice QFTs. However, let $\Phi_{0, n}$ denote the corresponding Q-map, and let $P_{n}: V_{0} \rightarrow V_{0, n}$ be the canonical projection, i.e. $P_{n}\left(\left(c_{m}\right)\right)=\left(c_{1}, \ldots, c_{n}, 0,0, \ldots\right)$ for $\left(c_{m}\right) \in V_{0}$, then $\Phi_{0, n}=\Phi_{0} \circ P_{n}$ and $P\left(\Phi_{0, n}\right)$ is a sub-algebra of $P\left(\Phi_{0}\right)$. Since $P_{n}$ is a c-homomorphism, there exists a corresponding *homomorphism, $\pi_{n}: P\left(\Phi_{0}\right) \rightarrow P\left(\Phi_{0, n}\right)$, by proposition 1 so that $\Phi_{0, n}=\pi_{n} \circ \Phi_{0}$. Let $\left(\Phi_{0}, \omega\right)$ be a Q-theory, then $\omega_{(n)}=\left.\omega\right|_{P\left(\Phi_{0, n}\right)}$ defines a state on $P\left(\Phi_{0, n}\right)$, and $\left(\Phi_{0}, \omega_{(n)} \circ \pi_{n}\right)$ is a Q-theory. For the sake of notational convenience we denote $\omega_{(n)} \circ \pi_{n}$ simply by $\omega_{(n)}$ in the following. $\left(\Phi_{0}, \omega_{(n)}\right)$ represents a reduced system. We note that the series $\left(\omega_{(n)}\right)$ converges to $\omega$, and that the QFTs discussed in this paper can therefore be approximated on lattices, see also Sec. 6.1.

Theorem 3: Let $\left(\omega_{i}\right)$ be a net of states over $P\left(\Phi_{0}\right) .\left(\omega_{i}\right)$ converges to a state $\omega$ if and only if $\left(\omega_{i,(n)}\right)$ converges to $\omega_{(n)}$ for all $n \in$.

Proof: $\omega_{i,(n)} \rightarrow \omega_{(n)}$ is a consequence of $\omega_{i} \rightarrow \omega$. Let us assume that $\omega_{i,(n)} \rightarrow \omega_{(n)}$ for all $n \in$, and let $a \in P\left(\Phi_{0}\right)$. Since $a \in P\left(\Phi_{0, n}\right)$ for some $n \in$, and since for $n<m, V_{0, n} \subset V_{0, m}$ and $\omega_{(n)}=\left.\omega_{(m)}\right|_{P\left(\Phi_{0, n}\right)}$, the states $\omega_{(n)}$ defines a unique state $\omega$ on $P\left(\Phi_{0}\right)$.

In the remaining part of this paper, we will show that theorem 1 applies to many well-known QFTs, and that theorems 2 and 3 define the continuum limit of lattice QFTs in our approach. 


\section{Application to Wightman quantum field theories}

The relation of our approach to Wightman QFTs can be conveniently discussed with the help of the Wightman reconstruction theorem. For the sake of convenience, let us consider a hermitian scalar Wightman QFT. In the reconstruction theorem, Wightman QFTs are recovered as representations of a Borchers-Uhlmann algebra [1]. The test-function space in Wightman QFTs is Schwartz space, $S\left({ }^{d}\right)(d \geq 2)$. Using the terminology and the definitions of Sec. 2, the Borchers-Uhlmann algebra is given by $\mathcal{A}_{V}$ with $V=S\left({ }^{d}\right)$, and the corresponding Q-map is denoted by $\Phi_{V}: V \rightarrow \mathcal{A}_{V}$. Note that $S\left(^{d}\right)$ is separable, and that corollary 1 and theorem 1 apply.

Corollary 2: Let $(\Phi, \omega)$ be a hermitian scalar Wightman QFT, then $\Phi$ is a quotient of $\Phi_{V}\left(V=S\left(^{d}\right)\right)$, and $\Phi_{0}$ is a core for $\Phi$.

We note that corollary 2 applies to any (hermitian scalar) Wightman QFT involving $d \geq 2$ space-time dimensions, and to Wightman QFTs of interacting quantum fields as well as free quantum fields. Moreover, considering proposition 2 , we can re-formulate corollary 2 into a looser statement: There exists a ${ }^{*}$-homomorphism, $\pi$, so that $(\Phi, \omega)$ and $\left(\Phi_{0}, \omega \circ \pi\right)$ essentially yield the same quantum theory, which emerges from a representation of $P\left(\Phi_{0}\right)$.

Let us discuss two examples of Wightman QFTs in more detail. In general, the set of field operators in Wightman QFTs is irreducible. Moreover, let $V_{s}$ denote the subset of real functions in $S\left({ }^{d}\right)$, then the set $\left\{\Phi(f): f \in V_{s}\right\}$ is also irreducible, and $\Phi\left(V_{s}\right)$ generates $P(\Phi)$. In the usual Fock-space representation of free scalar fields [8], the operators $\Phi(f)\left(f \in V_{s}\right)$ are essentially self-adjoint, i.e. the QFT is regular. Unfortunately the situation is less straightforward for Wightman QFTs of interacting fields, since there do not exist that many examples. However, let us consider $P(\varphi)_{2}$ as presented in Ref. [9].

$P(\varphi)_{2}$ is defined in flat space-time with one time dimension and one space dimension. The corresponding Fock space, $\mathcal{F}$, of the free hermitian scalar QFT is the symmetric tensor algebra over $L_{2}()$. For each open bounded interval, $B \subset$, let $\mathcal{A}(B)$ denote the von-Neumann algebra generated by the operators $\exp \left(i \varphi\left(f_{1}\right)+i \pi\left(f_{2}\right)\right)\left(f_{1}, f_{2} \in C_{0}^{\infty}(B), f_{1}, f_{2}\right.$ real) and let $\mathcal{A}$ denote the norm closure of $\bigcup_{B} \mathcal{A}(B) . P(\varphi)_{2}$ is constructed by considering the GNS representation, $\left(H_{\omega}, \pi_{\omega}, \Omega_{\omega}\right)$, of $\mathcal{A}$ with respect to a specific state, $\omega$. In this representation, the unitary groups

$$
W_{t}\left(f_{1}, f_{2}\right)=\pi_{\omega}\left(\exp \left(i t \varphi\left(f_{1}\right)+i t \pi\left(f_{2}\right)\right)\right) \quad\left(f_{1}, f_{2} \in C_{0}^{\infty}(B)\right)
$$

are strongly continuous, and they have self-adjoint generators.

Let $\Phi(f)=\varphi(\operatorname{Re}(f))+i \pi(\operatorname{Im}(f))$, then $\Phi$ is a Q-map. Let $\Phi^{\prime}$ be the restriction of $\Phi$ to $C_{0}^{\infty}()$ (assuming the Schwartz-space topology), then $\Phi^{\prime}$ is a core for $\Phi$, and $\left(\Phi^{\prime}, \omega\right)$ is a regular Q-theory. Also, let $W_{s}=V_{s} \cap C_{0}^{\infty}()$, then the set $\left\{\Phi(f): f \in W_{s}\right\}$ is irreducible, i.e. $\mathcal{A} \subset P\left(\Phi^{\prime}\right)^{\prime \prime}=B\left(H_{\omega}\right)$. We can therefore say that $(\Phi, \omega)$ and $\left(\Phi^{\prime}, \omega\right)$ essentially yield the same quantum theories. Moreover, $\Phi_{0}$ is a core for $\Phi^{\prime}$, and proposition 2 applies.

\section{Application to free quantum field theories}

In this section, we discuss the relation of our general approach to free QFTs that implement canonical commutation relations (CCRs) or canonical anti-commutation relations (CARs) on Fock space. We first discuss both cases together without specifying if the Fock space, $\mathcal{F}$, is symmetric or anti-symmetric. We assume however that $\mathcal{F}$ is constructed over an infinite-dimensional, separable Hilbert space, $\mathcal{H}$.

In conventional representations [10], the annihilation and creation operators, $a(f)$ and $a^{*}(f)$, are both defined over $\mathcal{H}$, so that $a(f)$ is complex anti-linear and that $a^{*}(f)$ is complex linear. We note that $a(f)$ and $a^{*}(f)$ are densely defined, closed, and that $a(f)^{*}=a^{*}(f)$. If there is a complex conjugation, $C$, defined on $\mathcal{H}$, then $\mathcal{H}$ and $\mathcal{H}^{2}$ are testfunction spaces, and we can introduce the complex-linear Q-map $\Phi_{1}(f, g)=a^{*}(f)+a(C g)$. Since $\Phi_{1}(f, 0)=a^{*}(f)$ and $\Phi_{1}(0, C f)=a(f), P\left(\Phi_{1}\right)$ is the polynomial algebra generated by the irreducible set of operators $A=\left\{a(f), a^{*}(f)\right\}_{f \in \mathcal{H}}$. In particular, in the symmetric case (CCRs), we obtain

$$
\Phi(f)=\frac{a^{*}(f)+a(f)}{\sqrt{2}}=\frac{\Phi_{1}(f, 0)+\Phi_{1}(0, C f)}{\sqrt{2}} .
$$

Since $\mathcal{H}^{2}$ is separable, we can apply theorem 1.

Corollary 3: $\Phi_{0}$ is a core for $\Phi_{1}$.

The annihilation operators in free QFTs can conveniently be chosen as complex-linear operator-valued functionals if they are defined over the dual Hilbert space instead, i.e. one considers $a(f)$ with $f \in \mathcal{H}^{*}[6]$. One advantage of this choice is that vacuum expectation values become multi-linear functionals over the test-function space. However, by the Riesz lemma, there is a natural complex anti-linear bijection, $j: \mathcal{H} \rightarrow \mathcal{H}^{*}$, and we can define the corresponding complex conjugation on $W=\mathcal{H} \otimes \mathcal{H}^{*}$ by $C(f, g)=\left(j^{-1}(g), j(f)\right)$, i.e. $W$ is a test-function space (c.f. Sec. 2). In 
particular, let $W_{2}=l_{2}() \otimes l_{2}()^{*}$, and let $\Phi_{2}=\Phi_{W_{2}}$. For a separable Hilbert space, $\mathcal{H}$, choose a unitary operator $U: l_{2}() \rightarrow \mathcal{H}$, let $\bar{U}: \mathcal{H}^{*} \rightarrow l_{2}()^{*}$ denote the corresponding dual unitary operator, define $\Phi_{2}^{\prime}(f, g)=a^{*}(f)+a(g)$, and define $\pi_{U}\left(\Phi_{2}(f, g)\right)=\Phi_{2}^{\prime}(U f, \bar{U} g) . \pi_{U}$ is a ${ }^{*}$-homomorphism between $P\left(\Phi_{2}\right)$ and $P\left(\Phi_{2}^{\prime}\right)$, $\Phi_{2}^{\prime}$ is a quotient of $\Phi_{2}$, and the Fock representation is a ${ }^{*}$-homomorphic representation of $P\left(\Phi_{2}\right)$. We summarize.

Corollary 4: In any free QFT, the polynomial algebra generated by the irreducible set of operators $\left\{a(f), a^{*}(g)\right\}_{f \in \mathcal{H}, g \in \mathcal{H}^{*}}$ is *-homomorphic to $P\left(\Phi_{2}\right)$, and $\Phi_{0}$ is a core for $\Phi_{2}$.

Adopting the same loose language as after proposition 2, we state that free QFTs emerge from Fock representations of $P\left(\Phi_{0}\right)$.

Let us discuss two examples of free QFTs. In Ref. [11], CCRs are defined with $\mathcal{H}=L_{2}(S)$, where $S$ is a Cauchy surface of a globally hyperbolic manifold. Independence of the actual choice of $S$ is due to a *-isomorphism between representations of the CCRs over the same vector space $\left(C_{0}^{\infty}(M)\right)$. We note that a more detailed account is given in Ref. [6], where especially the arbitrariness of the choice of scalar product in the definition of $\mathcal{H}$ is discussed. However, we note that corollary 3 applies irrespective of the specifically chosen globally hyperbolic manifold and background metric, i.e. the QFTs emerge from representations of $P\left(\Phi_{0}\right)$ and $P\left(\Phi_{2}\right)$, respectively.

The second example are Dirac quantum fields on a globally hyperbolic manifold, $M$, that are constructed in Ref. [12]. The construction is based on the definition of a scalar product on $C_{0}^{\infty}(D S)$, where $D S$ is the Dirac spinor bundle of spinors on a Cauchy surface, $S$. $D S$ is a vector bundle, and $C_{0}^{\infty}(D S)$ is locally isomorphic to $C_{0}^{\infty}(S)^{4}$. The completion of $C_{0}^{\infty}(D S)$ yields a separable Hilbert space, $\mathcal{H}$. The dual Hilbert space, $\mathcal{H}^{*}$, is the closure of $C_{0}^{\infty}\left(D^{*} S\right)$, the space of cross sections with compact support over the dual vector bundle $D^{*} S$. The representation of CARs over $S$ is further defined as a representation of the CARs over the pair $\mathcal{H}, \mathcal{H}^{*}$. We note that corollary 4 applies irrespective of the specifically chosen globally hyperbolic manifold and background metric, i.e. the QFTs emerge from representations of $P\left(\Phi_{0}\right)$ and $P\left(\Phi_{2}\right)$, respectively.

\section{Application to perturbative quantum field theory in curved space- times}

The perturbative formulation of quantum theories of interacting fields in curved space-time is closely related to recent achievements in the field of algebraic QFT $[4,5]$. In conventional approaches (c.f. Sec. 4), free quantum fields in curved space-time are operator-valued distributions over a space of smooth, compactly-supported test functions on a specific space-time. Independence of the specific space-time background can be further achieved in a categorial approach [5], which is also applicable to perturbatively interacting quantum fields. Starting from free QFTs, renormalized perturbation theory on curved space-times was explicitely formulated by methods of microlocal analysis [13, 14, 15].

Let us consider the example of a QFT of scalar hermitian fields as it is presented in Ref. [14]. The first step is to enlarge the quantum-field algebra of the corresponding free QFT with the help of microlocal analysis. The test function space of a free scalar hermitian QFT on a globally hyperbolic space-time manifold, $M$, is $C_{0}^{\infty}(M)$. Assuming a quasi-free Hadamard state, we can represent the the quantum-field algebra by the GNS construction. In such a representation, however, the quantum fields are operator-valued distributions over a larger, distributional test-function space. Let

$$
W_{n}\left(x_{1}, \ldots, x_{n}\right)=: \phi\left(x_{1}\right) \ldots \phi\left(x_{n}\right): \omega
$$

denote the (Wick-ordered) operator-valued distribution that is defined over $C_{0}^{\infty}\left(M^{n}\right)$ and let $\omega$ denote a quasi-free Hadamard state $\left(n \geq 1, W_{0}=1\right)$. As can be shown by microlocal analysis, the operator-valued distributions are defined on a larger space, $E_{n}^{\prime}$, which contains $C_{0}^{\infty}\left(M^{n}\right)$ and which is a subspace of the dual space $C_{0}^{\infty}\left(M^{n}\right)^{\prime}$. Distributions in $E_{n}^{\prime}$ are compactly supported, and they satisfy the wave-front condition $(t) \subset G_{n}\left(t \in E_{n}^{\prime}\right)$, where $G_{n}=\left(T^{*} M\right)^{n} \backslash H_{n}$ and

$$
H_{n}=\left\{(x, k): x \in M, k \in\left(\bar{V}^{+}\right)^{n} \cup\left(\bar{V}^{-}\right)^{n}\right\}
$$

Note that such distributions can be multiplied with each other, so that local Wick polynomials can rigorously be defined. Let $\mathcal{W}$ be the ${ }^{*}$-algebra of operators generated by 1 and elements $\left\{W_{n}(t)\right\}_{n \in, t \in E_{n}}$ (c.f definition 2.1 in Ref. [14]). Note also that $E_{n}^{\prime}$ does not have a proper topology in the sense of Hörmander [16], but that it rather has a so-called pseudo topology, see Ref. [14] for more details.

However, let us endow $E_{n}^{\prime}$ with the sub-space topology, which is inherited from the weak* topology on $C_{0}^{\infty}\left(M^{n}\right)^{\prime}$. $E_{n}^{\prime}$ is separable, and complex conjugation is well-defined since $C_{0}^{\infty}\left(M^{n}\right)$ is a dense subspace, i.e. $E_{n}^{\prime}$ is a test-function space according to our definition in Sec. 2. Each $W_{n}$ is a Q-map, and the corresponding polynomial algebra, $P\left(W_{n}\right)$, is a sub-algebra of $\mathcal{W}$. As outlined in Sec. 2, we can combine the Q-maps by defining the test-function space $E^{\prime}=\bigoplus_{n} E_{n}^{\prime}$ and the Q-map $\Phi_{3}=\sum_{n} W_{n}$, so that we obtain $P\left(\Phi_{3}\right)=\mathcal{W}$. We apply theorem 1 .

Corollary 5: $\Phi_{0}$ is a core for $\Phi_{3}$. 
In the perturbative approach, interacting fields are formally defined by a perturbation series. Series of operators in $\mathcal{W}$ form an algebra $\mathcal{X}=\mathcal{W}$ with multiplication $\left(a_{n}\right) \star\left(b_{n}\right)=\left(a_{0} b_{0}, a_{1} b_{0}+a_{0} b_{1}, \ldots\right)$. Note that the product is defined as if one formally multiplies $\sum_{n} a_{n}$ and $\sum_{n} b_{n}$. Let us consider the test-function space $\bigoplus_{n} E^{\prime}$, on which we define the Q-map $\Phi_{4}(f)=\left(\Phi_{3}\left(f_{n}\right)\right)$. Using the CCRs, one can further show that $P\left(\Phi_{4}\right)$ contains all co-finite sequences in $\mathcal{X}$. Note that the co-finite sequences are dense in $\mathcal{X}$ if we consider a weak topology on $\mathcal{W}$ and the product topology on $\mathcal{X}$. However, we can again apply theorem 1.

Corollary 6: $\Phi_{0}$ is a core for $\Phi_{4}$.

Moreover, the interacting-field algebra is defined in Ref. [17] as a sub-algbera of $\mathcal{X}$ as follows. There exists a multi-linear map

$$
T_{L_{1}}^{(n)}: \mathcal{D}_{1}(M, \mathcal{V})^{n} \rightarrow \mathcal{X}
$$

where $\mathcal{D}_{1}(M, \mathcal{V})$ is a vector space and $L_{1}$ denotes the Lagrangian. $\mathcal{V}$ is a vector space, which is generated by a countably infinite (Hamel) basis, and $\mathcal{D}_{1}(M, \mathcal{V})$ is the space of compactly-supported smooth densities on $M$ with values in $\mathcal{V}$. An element $F \in \mathcal{D}_{1}(M, \mathcal{V})$ can be uniquely expressed as a finite sum, $F=\sum f_{i} v_{i}$, where $f_{i} \in C_{0}^{\infty}(M)$ and $v_{i} \in \mathcal{V}$. As a vector space, $\mathcal{D}_{1}(M, \mathcal{V})$ is therefore isomorphic to $\bigoplus_{n} C_{0}^{\infty}(M)$. The interacting-field algebra is further defined as the algebra generated by the images of the maps $T_{L_{1}}^{(n)}$.

Let us endow $\bigoplus_{n} C_{0}^{\infty}(M)$ with the topology induced by $C_{0}^{\infty}(M)$, and let the conjugation on $\bigoplus_{n} C_{0}^{\infty}(M)$ be induced by the usual complex conjugation on $C_{0}^{\infty}(M) \cdot \mathcal{D}_{1}(M, \mathcal{V})^{n}$ and $\left(\bigoplus_{m} C_{0}^{\infty}(M)\right)^{n}$ are hence test-function spaces for all $n \in$, and the maps $T_{L_{1}}^{(n)}$ satisfy our definition of a Q-map, see Sec. 2. Let $\Phi_{5}$ denote the Q-map combining the $T_{L_{1}}^{(n)}$, then the interacting-field algebra is $P\left(\Phi_{5}\right)$. We can again apply theorem 1 .

Corollary 7: $\Phi_{0}$ is a core for $\Phi_{5}$.

Moreover, in Ref. [18], an algorithm is presented to construct the Wilson operator-product expansion (OPE). The algorithm is generally applicable to perturbative interacting QFT in Lorentzian curved space-times, and it is explicitly presented for the example of a scalar hermitian self-interacting field. However, as proposed in Ref. [4], the OPE can actually be elevated to a fundamental level, so that the QFT is determined by its OPE. This yields a general axiomatic framework for QFTs in curved space-times. In particular, the algebra of interacting quantum fields, $\mathcal{F}_{i}$, is obtained by factoring the corresponding free algebra, $\mathcal{F}_{0}$, by a set of relations arising from properties of the OPE coefficients. These relations define an ideal, $I$, in $\mathcal{F}_{0}$, and $\mathcal{F}_{i}=\mathcal{F}_{0} / I$. We note that the corresponding quotient map, $\pi_{I}: \mathcal{F}_{0} \rightarrow \mathcal{F}_{i}$ is a ${ }^{*}$ homomorphism. As pointed out in this paper so far, we typically can find a ${ }^{*}$ homomorphism, $\pi_{0}: P\left(\Phi_{0}\right) \rightarrow \mathcal{F}_{0}$, so that $\pi_{0}\left(P\left(\Phi_{0}\right)\right)$ is dense in $\mathcal{F}_{0}$ with respect to an appropriate topology. Then, $\pi_{I} \circ \pi_{0}$ is a ${ }^{*}$ homomorphism that maps $P\left(\Phi_{0}\right)$ onto a dense set in $\mathcal{F}_{i}$ with respect to another topology. Adopting the same loose language as after proposition 2 , we then can state that a perturbative interacting QFT emerges from a representation of $P\left(\Phi_{0}\right)$, if the corresponding free QFT does.

\section{$6 \quad$ Further representations of $P\left(\Phi_{0}\right)$}

\subsection{Lattice quantum field theory}

Let us use quantum chromodynamics on a lattice (LQCD) as an example of a lattice QFT. LQCD is a nonperturbative approach to QCD. Calculations usually are performed using the Feynman path-integral approach. Starting point is a set of CCRs and CARs [19] for symmetric operators $x_{a}, p_{a}, \tilde{x}_{c}$, and $\tilde{p}_{c}$ :

$$
\begin{aligned}
{\left[q_{a}, p_{b}\right] } & =i \delta_{a, b}, \\
{\left[q_{a}, q_{b}\right] } & =\left[p_{a}, p_{b}\right]=0, \\
\left\{\tilde{q}_{c}, \tilde{p}_{d}\right\} & =i \delta_{c, d}, \\
\left\{\tilde{q}_{c}, \tilde{q}_{d}\right\} & =\left\{\tilde{p}_{c}, \tilde{p}_{d}\right\}=0 .
\end{aligned}
$$

The indexes represent the degrees of freedom of the quantum system, and they consist of a position, $x$, and a field index. In conventional QFT, $x$ formally is continuous, but in LQCD, $x$ is discrete and only takes finitely many values. In particular, the Feynman integrals are rigorously defined in LQCD, and calculations are performed in exactly the same way as in conventional QFT, where a space-time continuum is considered [20].

Let us assume that $a$ and $c$ take finitely many value, i.e. $1 \leq a \leq m$ and $1 \leq c \leq n$. Let $\mathcal{A}_{m, n}$ be the operator algebra generated by $\left\{x_{a}, p_{a}, \tilde{x}_{c}, \tilde{p}_{c}: 1 \leq a \leq m, 1 \leq c \leq n\right\}$. $\mathcal{A}_{m, n}$ is the operator algebra of the specific LQCD model instance. We define a function $x:{ }^{m} \rightarrow \mathcal{A}_{m, n}$ by $x\left(\left(\delta_{a b}\right)_{1 \leq b \leq m}\right)=x_{a}$ and by complex-linear extension, and we define $x\left(\left(z_{a}\right)\right)^{*}=x\left(\left(\bar{z}_{a}\right)\right) . x$ is a Q-map, and we analogously define the Q-maps $p, \tilde{x}$, and $\tilde{p}$. We further combine these Q-maps into one Q-map, $\Phi_{6, m, n}: V_{m, n} \rightarrow \mathcal{A}_{m, n}, V_{m, n}=^{m} \otimes^{m} \otimes^{n} \otimes^{n}=^{2 m+2 n}$. Since $V_{m, n}$ is finite-dimensional, we 
can apply theorem 1 .

Corollary 8: $\Phi_{6, m, n}$ is a quotient of $\Phi_{0}$.

In the continuum limit, the lattice spacing is supposed to approach 0, i.e. the indexes $a$ and $c$ take an increasing number of values $(n, m \rightarrow \infty)$. We note that this does not lead to truly continuous indexes, and that continuous indexes must be differently treated. However, let us assume that for each grid size a state $\omega_{m, n}$ is determined. Since $\Phi_{6, m, n}$ is a quotient of $\Phi_{0}$, we obtain a series of states over $P\left(\Phi_{0}\right)$. Let us denote the series by $\left(\omega_{j}\right)$ for the sake of notational convenience. Since $V_{m, n}=V_{0,2 m+2 n}$ and since $\omega_{j,(k)}=\left.\omega_{j,(2 n+2 m)}\right|_{P\left(\Phi_{0, k}\right)}(k \leq 2 n+2 m)$, we obtain a series of states, $\omega_{j,(k)}$, on each sub-algebra $P\left(\Phi_{0, k}\right)$ of $P\left(\Phi_{0}\right)$. If each series, $\left(\omega_{j,(k)}\right)$, converges on the corresponding sub-algebra $P\left(\Phi_{0, k}\right)$, then there exists a unique limit state $\omega$ by theorem 3 , and $\left(\Phi_{0}, \omega\right)$ is a Q-theory by theorem 2 . We note however that $\left(\Phi_{0}, \omega\right)$ is not necessarily a QFT, since one has to additonally ensure that essential physical features are retrieved in that limit.

Let us rephrase this result: Increasing grid sizes yield a series of states. Each state of a specific grid defines a so-called reduced state on each sub-grid. A necessary and sufficient criterion for a unique limit state in the continuum limit is that the series of reduced states converges on each sub-grid. If a limit state exists, then we obtain a Q-theory as defined in this paper, and if appropriate physical features are retrieved then the Q-theory is also a reasonable QFT.

\subsection{String theory}

We base our discussion of string theory on the lecture notes of R. J. Szabo [21]. String theory is still work in progress, and a thorough discussion of the relation of string theory to the approach in this paper is elusive so far. However, there are five different consistent formulations of string theory that are commonly seen as perturbative expansions of a unique underlying theory (M-theory), which is however not well understood yet. The five theories are related by dualities that map perturbative states in one theory to non-perturbative states in another theory.

However, the quantization of the bosonic string yields a countable set of raising and lowering operators that satisfy the relation $\left(a_{n}^{\mu}\right)^{*}=a_{-n}^{\mu}$, and, if closed strings are considered, $\left(\tilde{a}_{n}^{\mu}\right)^{*}=\tilde{a}_{-n}^{\mu}(0 \leq \mu \leq d, n \in)$. These operators satisfy CCRs. There are also zero-mode operators, $x_{0}^{\mu}$ and $p_{0}^{\mu}$, that are conjugate to each other and that also satisfy the CCRs. The operators act on a Fock space, and we can combine them into an equivalent set of self-adjoint operators as follows:

$$
\begin{aligned}
& x_{n}^{\mu}=\frac{a_{n}^{\mu}+a_{-n}^{\mu}}{2}, \quad p_{n}^{\mu}=\frac{i\left(a_{n}^{\mu}-a_{-n}^{\mu}\right)}{2} \\
& \tilde{x}_{n}^{\mu}=\frac{\tilde{a}_{n}^{\mu}+\tilde{a}_{-n}^{\mu}}{2}, \quad \tilde{p}_{n}^{\mu}=\frac{i\left(\tilde{a}_{n}^{\mu}-\tilde{a}_{-n}^{\mu}\right)}{2} .
\end{aligned}
$$

We assume that observables in bosonic string theory are contained in the closure of the operator algebra, $\mathcal{A}_{b}$, generated by the operators $\left\{x_{n}^{\mu}, p_{n}^{\mu}, \tilde{x}_{n}^{\mu}, \tilde{p}_{n}^{\mu}\right\}_{n \in}$ with respect to an appropriate topology. However, let $v_{n}=\left(\delta_{n, m}\right)_{m \in} \in V_{0}$ and let $x^{\mu}\left(v_{n+1}\right)=x_{n}^{\mu}$ and $p^{\mu}\left(v_{n+1}\right)=p_{n}^{\mu}$ for $n \in_{0}$, then, by complex-linear continuation, $x$ and $p$ define Q-maps over the test-function space $V_{0}$. Analogously we can define the additional Q-maps $\tilde{x}$ and $\tilde{p}$ if we consider closed strings.

Fermions further need to be included in string theory to avoid inconsistencies. Canonical quantization yields another countable set of operators satisfying $\left(\psi_{r}^{\mu}\right)^{*}=\psi_{-r}^{\mu}\left(r=0, \pm 1, \pm \frac{1}{2}, \pm 2, \pm \frac{3}{2}, \ldots\right)$. These operators generate the corresponding operator algebra $\mathcal{A}_{f}$, and we simply denote the total operator algebra generated by operators in $\mathcal{A}_{b}$ and $\mathcal{A}_{f}$ by $\mathcal{A}$. The Hilbert spaces of the various string theories are subspaces of the full Hilbert space that is obtained by canonical quantization, and there are corresponding representations of $\mathcal{A}$ on these sectors. As for the bosonic case, the fermionic operators can be combined into two Q-maps over $V_{0}$. So depending on the case, we obtain four to six Q-maps over $V_{0}$ that generate the operator algebra in string theory, $\mathcal{A}$. These Q-maps can be combined into one $\mathrm{Q}-\mathrm{map}, \Phi_{7, k}$, over the test-function space $V_{0}^{\otimes k}(k=4$ or $k=6)$, i.e. $P\left(\Phi_{7, k}\right)$ is the operator algebra in the respective string theory, and we can apply theorem 1 .

Corollary 9: $\Phi_{0}$ is a core for $\Phi_{7, k}$.

\section{REFERENCES}

[1] R. F. Streater and A. S. Wightman: PCT, Spin and Statistics, and All That. Princeton University Press, Princeton (2000)

[2] H.J. Borchers: On Structure of the Algebra of Field Operators. Nuovo Cimento 24, 1418-1440 (1962)

[3] A. Uhlmann: Über die Definition der Quantenfelder nach Wightman und Haag. Wiss. Zeitschr. Karl-Marx Univ. Leipzig $11,213-217(1962)$ 
[4] S. Hollands and R. M. Wald: Axiomatic quantum field theory in curved spacetime. Comm. Math. Phys. 293, 85-125 (2010)

[5] R. Brunetti, K. Fredenhagen, and R. Verch: The generally covariant locality principle: A new paradigm for local quantum physics. Comm. Math. Phys. 237, 31-68 (2003); see also K. Fredenhagen: Locally covariant quantum field theory. arXiv:hepth/0403007 (2004)

[6] R. M. Wald: Quantum Field Theory in Curved Spacetime and Black Hole Thermodynamics. The University of Chicago Press, Chicago (1994)

[7] O. Bratelli and D. W. Robinson: Operator Algebras and Quantum Statistical Mechanics I: C*-and W*-Algebras. Symmetry Groups. Decomposition of States. Springer, New York (1979)

[8] M. Reed and B. Simon: Methods of Modern Mathematical Physics II. Fourier analysis and self-adjointness. Academic Press, New York (1975).

[9] J. Glimm and A. Jaffe: The Energy Momentum Spectrum and Vacuum Expectation Values in Quantum Field Theory, II. Comm. Math. Phys. 22, 1-22 (1971)

[10] O. Bratelli and D. W. Robinson: Operator Algebras and Quantum Statistical Mechanics II: Equilibrium States. Models in Quantum Statistical Mechanics. Springer, New York (1981)

[11] J. Dimock: Algebras of Local Observables on a Manifold. Comm. Math. Phys. 77, 219-228 (1980)

[12] J. Dimock: Dirac quantum fields on a manifold. Trans. Am. Math. Soc. 269, 133-147 (1982)

[13] R. Brunetti and K. Fredenhagen: Microlocal Analysis and Interacting Quantum Field Theories: Renormalization on Physical Backgrounds. Comm. Math. Phys. 208, 623-661 (2000)

[14] S. Hollands and R. M. Wald: Local Wick Polynomials and Time-Ordered-Products of Quantum Fields in Curved Spacetime. Comm. Math. Phys. 223, 289-326 (2001)

[15] S. Hollands and R. M. Wald: Existence of Local Covariant Time Ordered Products of Quantum Fields in Curved Spacetime. Comm. Math. Phys. 231, 309-345 (2002)

[16] L. Hörmander: The Analysis of Linear Partial Differential Operators I. Springer, Berlin (1985)

[17] S. Hollands and R. M. Wald: On the Renormalization Group in Curved Spacetime. Comm. Math. Phys. 237, 123-160 (2003)

[18] S. Hollands: The operator product expansion for perturbative quantum field theory in curved spacetime. J. Math. Phys. 273, 1-36 (2007)

[19] S. Weinberg: The Quantum Theory of Fields I. Foundations. Cambridge University Press, Cambridge (1995)

[20] R. Gupta: Introduction to Lattice QCD. arXiv:hep-lat/9807028 (1998)

[21] R. J. Szabo: Busstep lectures on string theory. arXiv:hep-th/0207142 (2002) 